\title{
Metodología para el análisis y diseño de circuitos con transistores y diodos en asignaturas de electrónica utilizando una herramienta computacional
}

\section{Methodology for the analysis and design of circuits with transistors and diodes in electronics courses using a computational tool}

\author{
Luis Eduardo Ramírez-Carvajala ${ }^{a}$ Karla Cecilia Puerto-López ${ }^{\mathrm{b}}$ Luilly Alejandro García-Ortiz ${ }^{\mathrm{c}}$ \\ angeniero Electrónico, luiseduardorc@ufps.edu.co, https://orcid.org/0000-0002-2253-9221, Universidad Francisco de Paula Santander, Cúcuta, \\ Colombia \\ bMagister en Ingeniería de Telecomunicaciones, karlaceciliapl@ufps.edu.co, https://orcid.org/0000-0003-3749-676X, Universidad Francisco de \\ Paula Santander, Cúcuta, Colombia \\ 'Doctorando en Ingeniería Eléctrica, luillygarcia@ufmg.br, https://orcid.org/0000-0001-5167-2585, Universidad Federal de Minas Gerais, Belo \\ Horizonte, Brasil
}

Forma de citar: Ramírez-Carvajal, L. E. ., Puerto-López, K. C. ., \& García-Ortiz, L. A. . (2021). Metodología para el análisis y diseño de circuitos con transistores y diodos en asignaturas de electrónica utilizando una herramienta computacional. Eco Matemático, 12 (2), 14-24

Recibido: 5 de febrero de 2021

Aceptado:8 de abril de 2021

\section{Palabras clave \\ Herramienta, tecnológica, análisis, diseño, electrónica}

\section{Keywords \\ Tool, technological, analysis, design, electronic}

\begin{abstract}
Resumen: Las herramientas tecnológicas mejoran el rendimiento académico haciendo más dinámicos y eficientes los procesos de enseñanza y aprendizaje. Se presenta una metodología de análisis y diseño de circuitos mediante el uso de una herramienta tecnológica en asignaturas de electrónica de la Universidad Francisco de Paula Santander. Se desarrolló una metodología ágil que consistió en cuatro elementos: planeación, diseño, codificación y prueba. En la planeación se consultaron las fuentes adecuadas y se desarrolló una metodología de análisis y diseño de circuitos con diodos y transistores. En el diseño se utilizó diagramación UML para el modelado del software. En codificación se utilizó lenguaje de programación java y se realizó una interfaz dinámica con la herramienta NetBeans. Finalmente se elaboraron pruebas a la interfaz, que consistieron en llevar a cabo diseños y análisis de circuitos con diodos y transistores, que fueron luego modelados en el software de simulación QUCS para calcular los errores en las variables y avalar la herramienta desarrollada. Los errores en todas las pruebas fueron inferiores al $2 \%$, lo que implica que la interfaz es eficiente, interactiva e incentiva los procesos de enseñanza y aprendizaje haciéndolos más dinámicos.
\end{abstract}

\begin{abstract}
Technological tools improve academic performance by making teaching and learning processes more dynamic and efficient. A methodology for the analysis and design of circuits through the use of a technological tool in electronics courses at the Universidad Francisco de Paula Santander is presented. An agile methodology was developed consisting of four elements: planning, design, coding and testing. In planning, appropriate sources were consulted and a methodology for analysis and design of circuits with diodes and transistors was developed. In design, UML diagramming was used for software modeling. In coding, Java programming language was used and a dynamic interface was created with the NetBeans tool. Finally, the interface was
\end{abstract}

*Autor para correspondencia: karlaceciliap1@ufps.edu.co

https://doi.org/10.22463/17948231.3075

2462-8794(C) 2021 Universidad Francisco de Paula Santander. Este es un artículo bajo la licencia CC BY 4.0 
tested, which consisted of carrying out designs and analysis of circuits with diodes and transistors, which were then modeled in QUCS simulation software to calculate the errors in the variables and endorse the developed tool. The errors in all tests were less than $2 \%$, which implies that the interface is efficient, interactive and encourages teaching and learning processes, making them more dynamic.

\section{Introducción}

Mucho se menciona sobre cual elemento en particular revolucionó la tecnología históricamente. Pero es la electrónica en su conjunto el invento más importante; sin ella, el hombre moderno no habría logrado el actual desarrollo científico y tecnológico, J. C. A Floriani (2006), pues ha permeado muchas áreas del conocimiento permitiendo que se adelanten diversas investigaciones en campos como: visión artificial, C. V. Niño Rondón, B. Medina Delgado, J. J. Ramírez Mateus, S. Alexander Castro, D. Guevara Ibarra y K. C. Puerto López (2020), energía solar, A. G. Vera Dávila, J. C. Delgado Ariza y S. B. Sepúlveda Mora (2018), nanomedicina, S. A. Torres Pérez, C. E. Torres Pérez, M. Pedraza Escalona, S. M. Pérez Tapia y E. Ramón Gallegos (2020), radiocomunicaciones, A. A. Baez Perez y Á. J. Soto Vergel (2019), Computación cuántica J. Sánchez Mondragón, A. Molina, S. Sánchez Sánchez y M. Torres Cisneros (2005), entre otros.

Al ser una ciencia en constante desarrollo debido a la creciente evolución de sus tendencias por efecto de la globalización, Z. V. Arévalo y A. Hernández F (2007), ha provocado que la enseñanza de la misma sea abordada mediante diversas estrategias de aprendizaje las cuales están relacionadas positivamente con el desempeño académico $\mathrm{J}$. J. Ramírez Echeverry, . F. A. Olarte Dussan y À. García Carrillo (2007). Clases magistrales, talleres, laboratorios, desarrollo de proyectos y visitas empresariales, son algunas de las metodologías tradicionales utilizadas C. L. Garzón Castro, L. M. Beltrán Sierra y P. Martínez Sánchez (2009). Sin embargo, es necesario contar con el carácter innovador de la tecnología dentro de los procesos académicos porque tienen un impacto positivo como estrategia que afianza la comunicación entre docentes y alumnos, N. Arellano, J. Fernandez, M. V. Rosas y M. E. Zuñiga (2014).

Son muchas las bondades del uso de las herramientas tecnológicas dentro de los procesos académicos e ingenieriles, como por ejemplo, la interactividad y el dinamismo en el diseño de circuitos asistido por computadora L. E. Ramírez Carvajal, G. A. Sierra Peñarada, K. C. Puerto López y D. Guevara Ibarra (2019), la efectividad en procesos de caracterización de dispositivos S. A. Castro Casadiego, C. V. Niño Rondón y B. Medina Delgado (2020), la mejora del rendimiento académico estudiantil, J. A. Zamora Araya, J. Ramírez Jiménez y F. Delgado Navarro (2020), la optimización del aprendizaje mediante la implementación de cursos interactivos A. Ávila, E. Flórez Serrano y O. Guladrón (2012), la eficacia de los análisis matemáticos computacionales L. E. Ramírez Carvajal, K. C. Puerto López y J. Ramírez Mateus (2020), entre otros.

\section{Estado del arte}

M.J.Rizales Semprum, C.L. Gómez Valderrama y C. A. Hernández Suarez (2019), determinaron en los docentes de ciencias en un liceo Bolivariano del estado Trujillo - Venezuela que un grupo significativo de ellos desconocían la plataforma tecnológica utilizada en la modalidad de educación interactiva a distancia y su utilidad en el proceso de enseñanza aprendizaje. Por otra parte, E. F. Camelo Quintero (2019), demostró que en un cuso de instrumentación el laboratorio simulado de manera virtual no fue equivalente al laboratorio presencial debido a que los simuladores desprecian situaciones críticas o fenómenos que pueden generar un pobre desarrollo de las competencias de los estudiantes. 
Sin embargo, T. Rojano (2003), describe que un proyecto de incorporación de entornos tecnológicos de aprendizaje en la enseñanza de las matemáticas y las ciencias en la escuela secundaria pública desarrollado en México por iniciativa de la secretaria de educación pública y el Instituto Latinoamericano de la Comunicación Educativa arrojó excelentes resultados, los cuales han servido de referencia para la incorporación explícita al currículo de otros países del uso de entornos tecnológicos de aprendizaje, como el de la hoja electrónica de cálculo para la resolución de problemas aritmético-algebraicos y para la modelación matemática en ciencias. Así mismo, M. L. García Rodríguez, A. H. Ortiz García y J. E. Velázquez (2020), afirman que con la difusión de las tecnologías se han ido posicionando varias líneas de investigación: análisis de los cambios experimentados en el currículum de matemáticas como consecuencia de la difusión de las nuevas tecnologías; enseñanza de temas matemáticos en cursos de informática; diseño y uso de herramientas TIC como mediadores de procesos de enseñanza $\mathrm{y}$ aprendizaje en matemáticas y estudio del proceso educativo en matemáticas en entornos de aprendizaje basados en tecnología. De igual forma, I. J. Cacua Peñaloza (2018), propuso un recurso instruccional apoyado en la realidad aumentada para el desarrollo de los contenidos de estructuras del área de tecnología en estudiantes de séptimo grado, cuya investigación logró establecer que los docentes emplean estrategias didácticas poco adecuadas en el desarrollo de los contenidos de estructuras, de igual manera, es necesario sostener que las herramientas y recursos que desde la tecnología promueven el empleo de la realidad aumentada, son significativas, aunque no son empleadas por los docentes de manera adecuada, de igual forma se logró establecer que existe una muy alta factibilidad de aplicación de un recurso instruccional apoyado en la realidad aumentada para el desarrollo de los contenidos de estructuras del área de tecnología en los estudiantes. Finalmente Cuicas et al. M. Cuicas Avila, E. Debel Chourio, L. Casadei Carniel y Z. Alvarez Vargas (2007), llevaron a cabo una investigación que tuvo como propósito el desarrollo de habilidades del pensamiento y el mejoramiento del aprendizaje en alumnos y alumnas de la asignatura Matemática II, del Decanato de Ingeniería Civil mediante el empleo de estrategias instruccionales basadas en el uso del software matemático. Los datos recogidos se analizaron utilizando estadística descriptiva. Los hallazgos del estudio fueron: (a) los conocimientos de los discentes mejoraron; y (b) estos pusieron en práctica sus procedimientos (sus habilidades cognoscitivas y metacognitivas). Por lo tanto, el estudio aportó evidencias para usar el software matemático bajo una metodología constructivista.

\section{Metodología}

Se desarrolla una metodología ágil, R. Pressman (2010), que consiste en cuatro elementos: planeación, diseño, codificación y prueba. Durante la planeación se consultan las fuentes y se eligen los temas a desarrollar. El diseño se lleva a cabo mediante diagramación UML (Lenguaje Unificado de Modelado). La codificación se desarrolla utilizando lenguaje de programación de uso libre. Y finalmente la prueba se realiza desde dos perspectivas: la evaluación del funcionamiento y la homologación de la herramienta. La metodología se ilustra en la Figura. 1. 


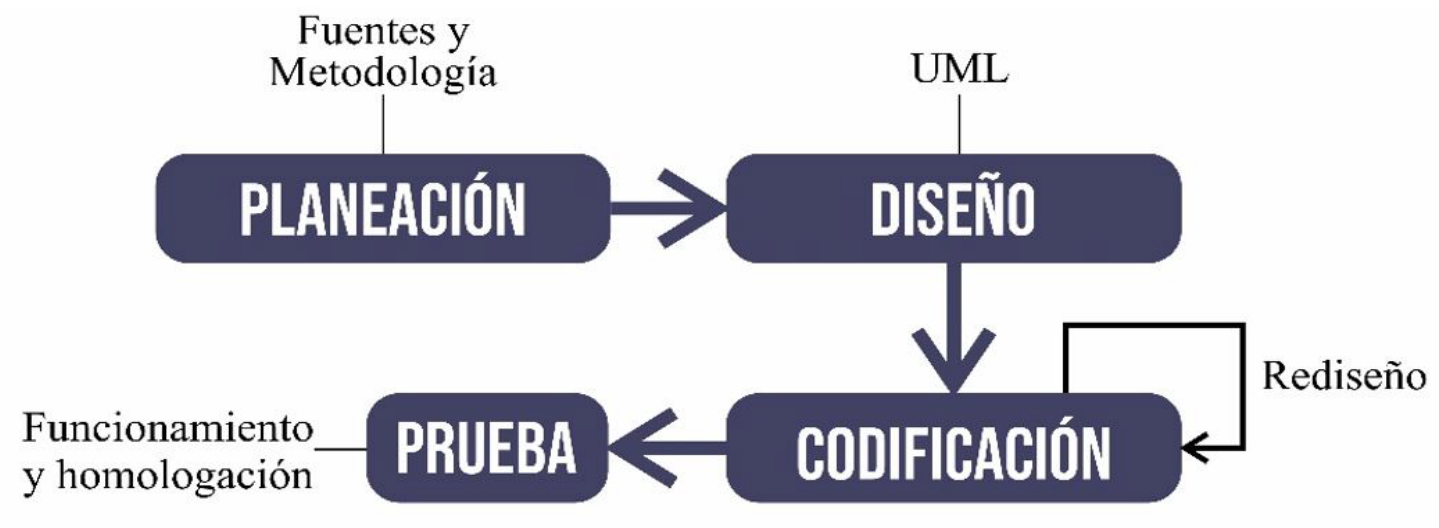

Figura 1. Metodología ágil desarrollada en la investigación. Fuente: Autores

\section{A.Planeación}

Se consultan los temas tratados en las asignaturas de electrónica del programa Ingeniería Electrónica de la universidad Francisco de Paula Santander, Universidad Francisco de Paula Santader, «Programa de Ingeniería electrónica,» Departamento de electricidad y electrónica, (2020). Se eligen los temas: diodos (perteneciente a la asignatura Electrónica 1), fuentes de corriente (perteneciente a la asignatura Electrónica 2), amplificadores de voltaje (perteneciente a la asignatura Electrónica 3) y reguladores de voltaje (perteneciente a la asignatura Electrónica de Potencia). Se consultan las fuentes, R. L. Boylestad y L. Nashelsky (2009), M. N. Horenstein (1997), A. R. Hambley (2001), A. Sedra y K. Smith (1998), C. J. Savant, M. Roden y G. Carpenter (1991), M. Rashid (1993), D. W. Hart (2001), para conocer los procedimientos de análisis y diseño existentes y se desarrolla la metodología de diseño y análisis.

\section{B. Diseño}

Con base en la metodología propuesta en planeación y mediante diagramación UML [31] se desarrolla la estructura funcional del sistema cuyo esquema general se observa en la Figura 2.

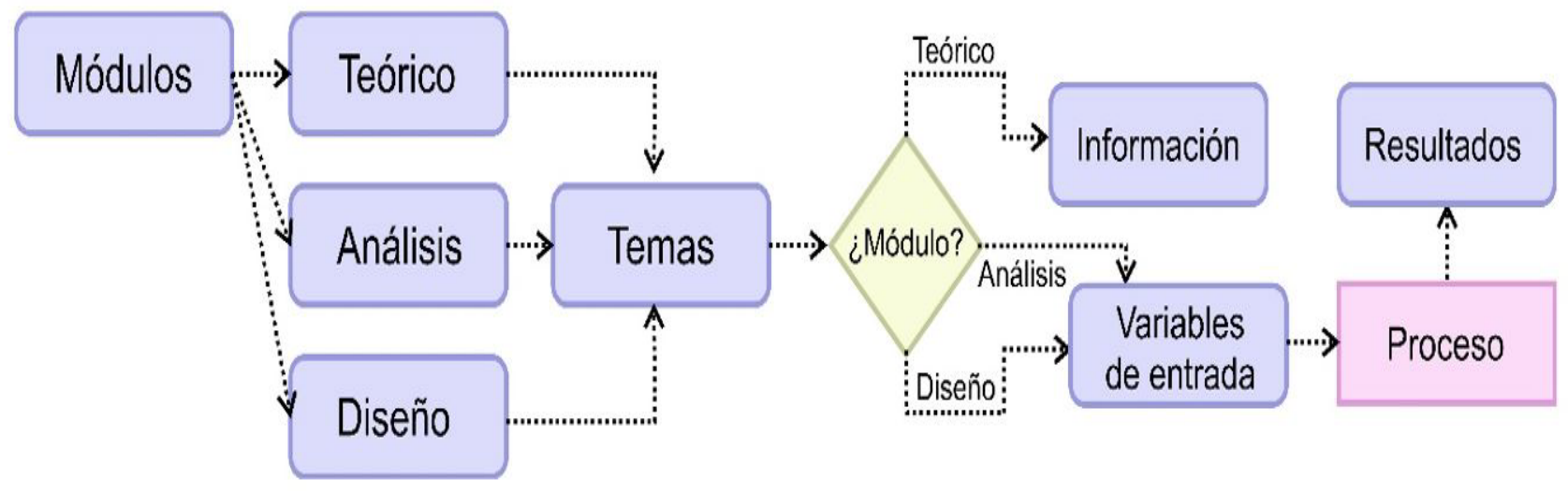

Figura 2. Esquema general del sistema de diagramación UML. Fuente: Autores

Rediseño es el proceso mediante el cual se cambia un sistema de software en forma tal que no altere el comportamiento externo del código, pero sí mejore la estructura interna. Es una manera disciplinada de limpiar el código (y modificar o simplificar el diseño interno) que minimiza la probabilidad de introducir 
errores. En esencia, cuando se rediseña, se mejora el diseño del código después de haber sido escrito, R. Pressman (2010).

\section{Codificación}

Mediante el uso de lenguaje de programación Java, J. M. Ladrón de Guevara (2015), se codifica el sistema y se desarrolla una herramienta interactiva utilizando el entorno de desarrollo NetBean,. En la Figura 3 se puede observar la interfaz desarrollada.

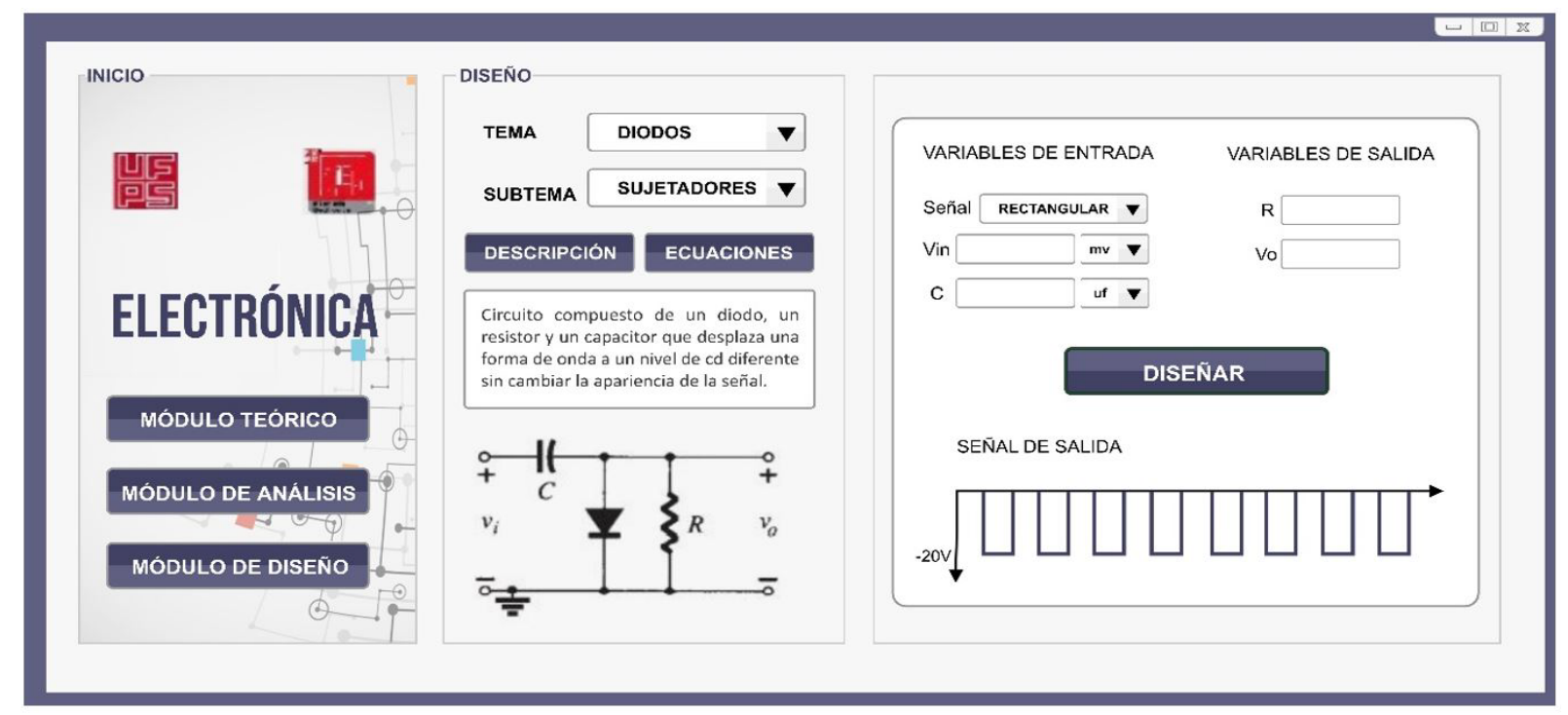

Figura 3. Interfaz desarrollada

Esta interfaz cuenta con una unidad de inicio y una unidad operativa. En la unidad de inicio se pueden encontrar tres botones: módulo teórico, módulo de análisis y módulo de diseño. Al seleccionar el módulo a trabajar la unidad operativa muestra la función a realizar.

Cuando se selecciona el módulo teórico la unidad operativa muestra información sobre los diferentes temas que conforman la herramienta: Definiciones, funciones, ecuaciones, esquemáticos y gráficas sobre los distintos temas que pueden ser elegidos mediante un seleccionador desplegable.

Cuando se selecciona el módulo de análisis o el módulo de diseño, la unidad operativa muestra dos secciones: una temática y otra procedimental. En la sección temática hay dos botones: "descripción" y "ecuaciones". Con el botón "descripción" se muestra una breve información sobre el circuito y el esquemático general. Y con el botón “ecuaciones” se muestra el procedimiento de análisis o diseño paso a paso. En la sección procedimental se introducen la variable de entrada y se desarrolla el proceso para finamente mostrar los resultados.

\section{Prueba}

Cuando se lleva a cabo un desarrollo iterativo, no existe una especificación del sistema que pueda ser utilizada para pruebas externas. Por consiguiente algunos enfoques para el desarrollo iterativo tienen un proceso de pruebas muy informal, I. Sommerville (2005). Para evitar algunos de estos problemas el método ágil utilizado en la investigación tiene un desarrollo basado en planificación, cuyas pruebas tienen dos enfoques. 
En un primer enfoque se realizan pruebas de funcionamiento y se evalúa la operatividad e interactividad de la interfaz. Se utiliza la herramienta para llevar a cabo un proceso de análisis y un proceso de diseño por cada configuración posible según las temáticas incorporadas al proyecto. En esta primera fase se desarrollan esquemáticos de rectificadores, recortadores y sujetadores con diodos, de fuentes espejo, simples, widlar, Wilson y Cascode, de amplificadores de voltaje con transistores bjt y jfet y de reguladores serie, paralelo, reductores, elevadores e inversores.

En un segundo enfoque de homologación se toman los esquemáticos realizados, se modelan en el software de simulación QUCS, M. E. Brinson y S. Jahn (2009) y se mide el error entre las dos herramientas en las diferentes variables de los circuitos. Esto se realiza con el propósito de validar la herramienta desarrollada.

\section{Resultados y discusión}

En la Figura 4 se observa un ejemplo de un circuito con diodos desarrollado en la herramienta y modelado en el simulador. El esquemático muestra un rectificador de media onda producto de un proceso de diseño.

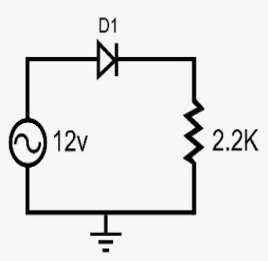

En herramienta tecnológica

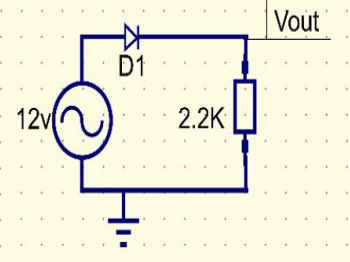

En software de simulación
Figura 4. Esquemático, rectificador de media onda. Fuente: Autores

En la Figura 5 se observa la señal de salida del rectificador de media onda, tanto en la herramienta tecnológica como en el simulador.

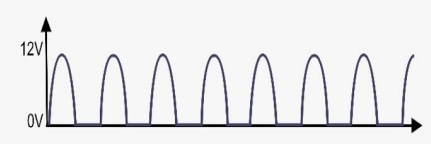

En herramienta tecnológica

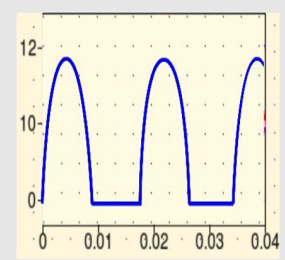

En software de simulación
Figura 5. Señal de salida, rectificador de media onda. Fuente: Autores.

En la Figura 6 se observa un ejemplo de un circuito de una fuente de corriente desarrollado en la herramienta y modelado en el simulador. El esquemático muestra una configuración Widlar producto de un proceso de análisis.

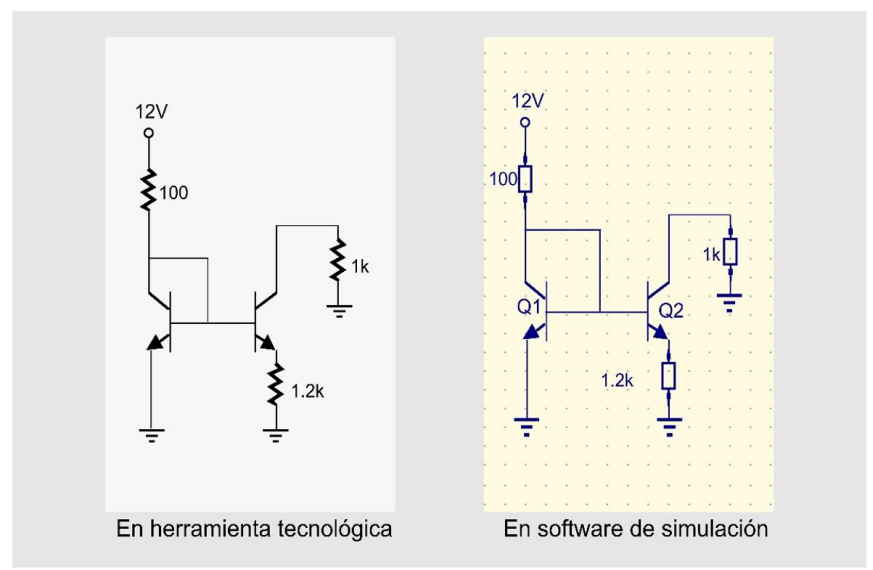

Figura 6. Esquemático, fuente de corriente Widlar. Fuente: Autores

En la Figura 7 se observa la corriente de salida de la fuente Widlar, tanto en la herramienta tecnológica como en el simulador.

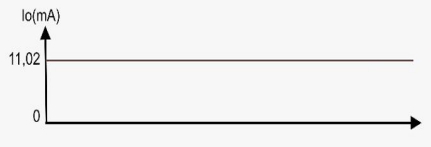

En herramienta tecnológica

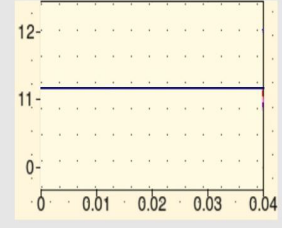

En software de simulación
Figura 7. Corriente de salida, fuente Widlar. Fuente: Autores

En la Figura 8 se observa un ejemplo de un circuito amplificador de voltaje desarrollado en la herramienta y modelado en el simulador. El 
esquemático muestra dos etapas, una con transistor bjt y otra con transistor jfet, producto de un proceso de diseño.

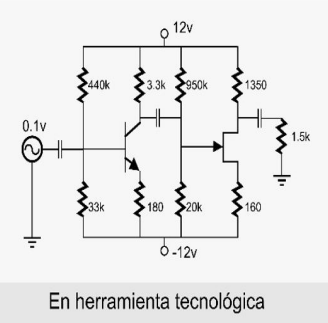

Figura 8. Esquemático, amplificador de voltaje 2 etapas. Fuente: Autores

En la Figura 9 se observa la señal de salida del amplificador 2 etapas, tanto en la herramienta tecnológica como en el simulador.
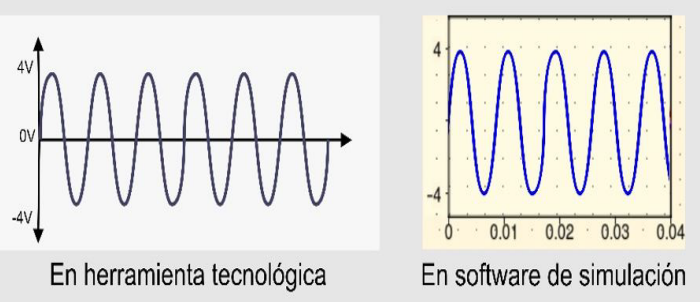

Figura 9. Adquisición y modificaciones iniciales a la imagen. Fuente: Autores

En la Figura 10 se observa un ejemplo de un circuito desarrollado en la herramienta y modelado en el simulador. El esquemático muestra un regulador de voltaje producto de un proceso de análisis.

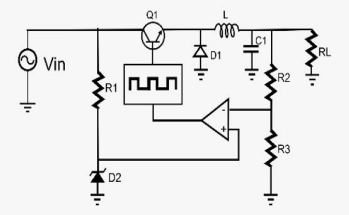

En herramienta tecnológica

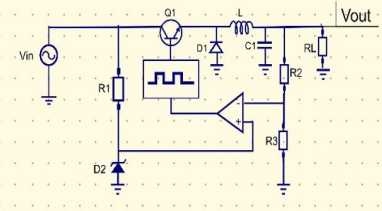

En software de simulación

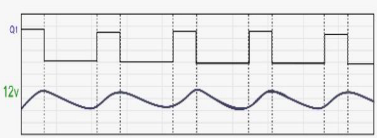

En herramienta tecnológica

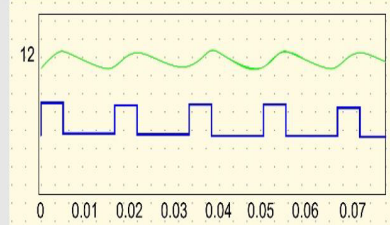

En software de simulación
Figura 11. Señal de salida, regulador de voltaje. Fuente: Autores

En la tabla I se muestran los errores promedio para todos los circuitos desarrollados en la herramienta e implementados en el software de simulación. Se compara para cada circuito: las corrientes, los voltajes y las señales de salida y se calcula el error promedio en todas las variables evaluadas.

Figura 10. Esquemático, regulador de voltaje. Fuente: Autores

En la Figura 11 se observa la señal de salida del regulador de voltaje, tanto en la herramienta tecnológica como en el simulador. 
Tabla I. Errores promedio en variables evaluadas

\begin{tabular}{|c|c|c|c|c|}
\hline Tema & Configuración & $\begin{array}{l}\text { Errores en } \\
\text { corrientes }\end{array}$ & $\begin{array}{l}\text { Errores en } \\
\text { voltajes }\end{array}$ & $\begin{array}{c}\text { Errores en } \\
\text { señales de salida }\end{array}$ \\
\hline \multirow{4}{*}{ Circuitos con diodos } & $\begin{array}{l}\text { Rectificador de } \\
\text { media onda }\end{array}$ & 0.871 & 1.231 & 0.888 \\
\hline & $\begin{array}{l}\text { Rectificador de onda } \\
\text { completa }\end{array}$ & 1.163 & 1.444 & 1.563 \\
\hline & Recortadores & 0.731 & 1.565 & 1.782 \\
\hline & Sujetadores & 0.576 & 1.452 & 0.472 \\
\hline \multirow{5}{*}{ Fuentes de corriente } & Espejo & 1.235 & 1.332 & 1.588 \\
\hline & Simples & 1.675 & 1.938 & 1.972 \\
\hline & Widlar & 1.896 & 1.397 & 1.190 \\
\hline & Wilson & 1.684 & 0.999 & 1.009 \\
\hline & Cascode & 1.264 & 0.889 & 1.279 \\
\hline \multirow{3}{*}{$\begin{array}{l}\text { Amplificadores } \\
\text { multietapa }\end{array}$} & $\begin{array}{l}\text { Amplificadores con } \\
\text { transistores bjt }\end{array}$ & 0.231 & 0.374 & 0.936 \\
\hline & $\begin{array}{l}\text { Amplificadores con } \\
\text { transistores jfet }\end{array}$ & 0.989 & 1.242 & 0.392 \\
\hline & $\begin{array}{l}\text { Amplificadores con } \\
\text { transistores bit y jfet }\end{array}$ & 0.862 & 1.252 & 1.293 \\
\hline \multirow{5}{*}{ Regladores de voltaje } & Serie & 0.729 & 0.472 & 1.557 \\
\hline & Paralelo & 1.283 & 1.384 & 0.835 \\
\hline & Reductores & 0.286 & 1.113 & 1.283 \\
\hline & Elevadores & 0.297 & 1.341 & 0.924 \\
\hline & Inversores & 1.236 & 0.989 & 1.993 \\
\hline
\end{tabular}

Fuente: Autores

Se involucran a la investigación 4 temáticas: diodos, fuentes de corriente, amplificadores multietapa y reguladores de voltaje. De dichas temáticas se toman 17 configuraciones para las cuales se hace una prueba de análisis y una de diseño. En total se desarrollan 34 circuitos que son modelados en el software de simulación y finalmente como lo muestra la Tabla 1, se evalúan corriente, voltajes y las señales de salida. Se observa que ninguno de los errores es superior al $2 \%$.

\section{Conclusiones}

Las metodologías desarrolladas para llevar a cabo procesos matemáticos complejos dentro del campo de la ingeniería deben estar estructuradas con enfoques específicos de acuerdo al propósito planteado como se evidencia en la investigación, teniendo en cuenta que la metodología implementada fue acertada para el desarrollo de la herramienta cumpliendo con el propósito del proyecto. No basta simplemente con ejecutar ecuaciones matemáticas de manera mecánica y desarrollar procedimientos analíticos que cumplen con requerimientos algebraicos sin tener en cuenta el enfoque metodológico que en el caso presente tiene un contexto pedagógico.

La herramienta tecnológica desarrollada contiene en su sistema no solamente un procedimiento matemático que se ejecuta para el desarrollo de un tema específico en ingeniería electrónica sino que busca desempeñar un papel pedagógico y experimental. Es una herramienta integral que permite llevar a cabo procesos de aprendizaje mediante el módulo teórico, procesos que afianzan el conocimiento mediante el módulo de análisis y procesos experimentales siendo útil en dicho campo mediante el módulo de diseño, teniendo en cuenta que puede ser un complemento de laboratorio para el desarrollo de prácticas en las diferentes temáticas incorporadas. 
La herramienta desarrollada es asertiva, pues los errores en todas las variables evaluadas fueron siempre inferiores al $2 \%$ lo que permite afianzar e incentivar el buen desempeño de dichas herramientas dentro de los procesos académicos, motivando al uso y desarrollo de nuevas tecnología que permitan optimizar la enseñanza y el aprendizaje en las distintas áreas del conocimiento.

La electrónica da pasos agigantados hacia un futuro tecnológico que cada día es más sorprendente, enfocando su desarrollo en la parte matemática, ingenieril y técnica. Sin embargo es necesario involucrar en dicho avance los procesos cognitivos y metodológicos para fortalecer la labor pedagógica e involucrar cada día más en la revolución científica que se avecina uno de los pilares fundamentales del conocimiento: la investigación.

\section{Referencias}

A. A. Baez Perez y Á. J. Soto Vergel, «Enseñanza de sistemas de radiocomunicaciones terrestres con línea de vista mediante software educativo,» Educación en Ingeniería, vol. 14, n 28 , pp. 7887, 2019

A. Ávila, E. Flórez Serrano y O. Guladrón, «Diseño e implementación de un curso interactivo multimedia para el aprendizaje de los procesos CAM en un centro de mecanizado Leadwell V30,» Revista Colombiana de Tecnología de Avanazada, vol. 2, no 20, pp. 50-56, 2012

A. G. Vera Dávila, J. C. Delgado Ariza y S. B. Sepúlveda Mora, «Validación del modelo matemático de un panel solar empleando la herramienta Simulink de Matlab,» Investigacón, desarrollo e innovación, vol. 8, $\mathrm{n}^{\circ} 2$, pp. 343356,2018

A. R. Hambley, Electrónica, Pearson Educación, 2001

A. Sedra y K. Smith, Microelectronic Circuits, Oxford University, 1998

C. L. Garzón Castro, L. M. Beltrán Sierra y P. Martínez Sánchez, «Estudio de percepción sobre metodologías de enseñanza de temas de electrónica en programas diferentes a ingeniería electrónica,» Educación en ingeniería, no 8, pp. 93-101, 2009

C. V. Niño Rondón, B. Medina Delgado, J. J. Ramírez Mateus, S. Alexander Castro, D. Guevara Ibarra y K. C. Puerto López, «Comparación multiplaforma de técnicas basadas en visiónartificial para detección de personas en espacios abiertos,» Investigación e innovación en ingenierías, vol. 9, n 1 , pp. 2233, 2020

C. J. Savant, M. Roden y G. Carpenter, Diseño Electrónico, Circuitosy Sistemas, Massachusetts: Addison-Wesley, 1991

D. W. Hart, Electrónica de potencia, Madrid: Pearson Educacion. S. A. , 2001 NetBeans, «NetBeans,» [En línea]. Available: https://netbeans.org/

E. F. Camelo Quintero, «Implementación de prácticas de laboratorio en la educación virtual de los programas de ingeniería electrónica y telecomunicaciones,» Revista Virtualmente, vol. 7, no 1, pp. 29-44, 2019

I. J. Cacua Peñaloza, «Recurso instruccional para el desarrollo del contenido de estructuras mediante la aplicación de la realidad aumentada en el área de tecnología. Caso: colegio "Pablo Correa León" Cúcuta, Colombia,» Eco matemático, vol. 9, no 1, pp. 97-121, 2018

I. Sommerville, Ingeniería del Software, 7 ed., Madrid: Pearson Eduacion, S.A., 2005

J. A. Zamora Araya, J. Ramírez Jiménez y F. Delgado Navarro, «Uso de herramientas tecnológicas y su impacto en el rendimiento en el curso de Cálculo II de la Universidad Nacional,» Eco Matemático, vol. 11, nº 1, pp. 6-17, 2020

J. C. A Floriani, «Sobre la historia de la Electrónica en el primer centenario de su nacimiento: la era Termoiónica,» IEEE Latin America Transactions, vol. 4, $\mathrm{n}^{\mathrm{o}} 4$, pp. 242-248, Junio 2006

J. J. Ramírez Echeverry, . F. A. Olarte Dussan y À. García Carrillo, «Estrategias de aprendizaje usadas por estudiantes de ingeniería eléctrica e ingeniería electrónica de primer semestre,» 
Educación en ingeniería, vol. 9, n 18 , pp. 216227, 2014

J. M. Ladrón de Guevara, Fundamentos de programación en Java, Madrid: Editorial EME, 2015

J. Rumbaugh, I. Jacobson y G. Booch, El lenguaje unificado de modelado, manual de referencia, Madrid: Pearson Educacion, 2000

J. Sánchez Mondragón, A. Molina, S. Sánchez Sánchez y M. Torres Cisneros, «Comparison of the Dicke Model and the Hamiltonian for n Quantum Dots,» de Quantum Dots, Nanoparticles, and Nanoclusters II, 2005

L. E. Ramírez Carvajal, G. A. Sierra Peñarada, K. C. Puerto López y D. Guevara Ibarra, «Computeraided design software for multi-stage amplifiers with bipolar transistors and field effect,» Journal of Physics: Conference Series, vol. 1418, $\mathrm{n}^{\mathrm{o}} 1$, p. 7, 2019

L. E. Ramírez Carvajal, K. C. Puerto López y J. Ramírez Mateus, «Mathematical analysis of physical stability in the design of bipolar amplifiers through a computer tool,» Journal of Physics: Conference Series, vol. 1672, n ${ }^{\circ}$ 1, p. 6, 2020

M. Cuicas Avila, E. Debel Chourio, L. Casadei Carniel y Z. Alvarez Vargas, «El software matemático como herramienta para el desarrollo de hablidades del pensamiento y mejoramiento del aprendizaje de las matemáticas,» Revista Electrónica Actualidades Investigativas en Educación, vol. 7, no 2, pp. 1-34, 2007

M. E. Brinson y S. Jahn, «Qucs: A GPL software package for circuit simulation, compact device modelling and circuit macromodelling from DC to RF and beyond,» International Journal of Numerical Modelling, Electronic Networks, Devices and Fields, vol. 22, nº 4, pp. 297-319, Julio 2009

M. J. Rizales Semprum, C. L. Gómez Valderrama y C. A. Hernández Suarez, «Uso de herramientas tecnológicas para la enseñanza de la ciencias en educación media diversificada de acuerdo a la modalidad de estudio a distancia,» Eco matemático, vol. 10, nº 2, pp. 35-46, 2019

M. L. García Rodríguez, A. H. Ortiz García y J. E. Velázquez, «La Investigación sobre el uso de tecnologías digitales en la enseñanza y el aprendizaje de las matemáticas: una revisión desde las memorias de los congresos en la última década,» Eco Matemático, vol. 11, nº 1, pp. 27 40,2020

M. N. Horenstein, Circuitos y Dispositivos Microelectrónicos, Prentice-Hall Hispanoamericana, 1997

M. Rashid, Electrónica de potencia, circuiros, dispositivos y aplicaciones, Prentice Hall Hispanoamericana, S. A., 1993

N. Arellano, J. Fernandez, M. V. Rosas y M. E. Zuñiga, «Estrategia metodológica de la enseñanza de la programación para la permanencia de los alumnos de primer año de Ingeniería Electrónica,» Revista Iberoamericana de Educación en Tecnología y Tecnología en Educación, $\mathrm{n}^{\mathrm{o}}$ 13, pp. 55-60, 2014

R. Pressman, Ingeniería del software, un enfoque práctico, 7 ed., México, D. F.: Mc Graw Hill, 2010

R. L. Boylestad y L. Nashelsky, Electrónica: Teoría de Circuitos y Dispositivos Electrónicos, Pearson Educación, 2009

S. A. Castro Casadiego, C. V. Niño Rondón y B. Medina Delgado, «Caracterización para la ubicación en la captura de video aplicado a técnicas de visión artificial en la detección de personas,» Revista Colombiana de Tecnología de Avanzada, vol. 2, n 36, pp. 83-88, 2020

S. A. Torres Pérez, C. E. Torres Pérez, M. Pedraza Escalona, S. M. Pérez Tapia y E. Ramón Gallegos, «Glycosylated Nanoparticles for Cancer-Targeted Drug Delivery,» Frontiers in Oncology, vol. 10, p. 10, 202

T. Rojano, «Incorporación de entornos tecnológicos de aprendizaje a la cultura escolar: proyecto de innovación educativa en matemáticas y ciencias,» Revista Iberoamericana de Educación, $\mathrm{n}^{\mathrm{o}}$ 33, pp. 135-165, 2003

Universidad Francisco de Paula Santader, «Programa 
de Ingeniería electrónica,» Departamento de electricidad y electrónica, 2020. [En línea]. Available: https://ww2.ufps.edu.co/ofertaacademica/ingenieria-electronica/995

Z. V. Arévalo y A. Hernández F, «Experiencias y reflexiones sobre la enseñanza de la Electrónica en tiempos de grandes cambios científicos, tecnológicos y de globalización,» IEEE Latin America Transactions, vol. 5, $\mathrm{n}^{\mathrm{o}}$ 1, pp. 62-69, 2007 